INPLASY

PROTOCOL

To cite: Teng et al. Perception and practices regarding ecigarettes among health sciences students and health professionals: protocol of a systematic review of world literature. Inplasy protocol 2020110065. doi:

10.37766/inplasy2020.11.0065

Received: 16 November 2020

Published: 16 November 2020

Corresponding author:

Cheong Lieng Teng

tengcl@gmail.com

Author Affiliation: International Medical University

Support: None.

Review Stage at time of this submission: Preliminary searches.

Conflicts of interest: None.

\section{Perception and practices regarding e-cigarettes among health sciences students and health professionals: protocol of a systematic review of world literature}

Teng, CL¹; Vasiwala, AB²; Bari, HR³; Khoo, EJ4.

Review question / Objective: What is the knowledge and attitude regarding e-cigarette, and usage of e-cigarette among health sciences students and health personnel around the world?

Condition being studied: E-cigarette emerged in 2017 and has shown somewhat uneven increase around the world due to the controversies generated and usage restriction imposed by various jurisdiction depending on ingredients used (some of which contain nicotine).

Information sources: An extensive literature search using a combination of mesh terms (Electronic Nicotine Delivery Systems) and text words (e-cigarette, electronic cigarette, vaping) will be carried out on electronic databases such as PubMed and Scopus. This will be supplemented by a Google Scholar search using the same search terms mentioned above. The year of publication of included studies will be from Jan 2017 to Dec 2020.

INPLASY registration number: This protocol was registered with the International Platform of Registered Systematic Review and Meta-Analysis Protocols (INPLASY) on 16 November 2020 and was last updated on 16 November 2020 (registration number INPLASY2020110065).

\section{INTRODUCTION}

Review question / Objective: What is the knowledge and attitude regarding $e-$ cigarette, and usage of e-cigarette among health sciences students and health personnel around the world?
Rationale: This study aims to document the variation in the perception and usage among the health sciences students and personnel around the world. 
Condition being studied: E-cigarette emerged in 2017 and has shown somewhat uneven increase around the world due to the controversies generated and usage restriction imposed by various jurisdiction depending on ingredients used (some of which contain nicotine).

\section{METHODS}

Search strategy: An extensive literature search using a combination of mesh terms (Electronic Nicotine Delivery Systems) and text words (e-cigarette, electronic cigarette, vaping) will be carried out on electronic databases using PubMed and Scopus. The search string that will be used is ("electronic nicotine delivery system" OR vaping OR e-cigarette OR "electronic cigarette") AND (health personnel OR doctor OR physician* OR nurses OR dentist* $^{*}$ OR pharmacist* OR students, medical OR students, dental OR students, nursing OR students, health occupations). This will be supplemented by a Google Scholar search using the same search terms mentioned above. The year of publication of included studies will be from Jan 2017 to Dec 2020.

Participant or population: Participants are health science students (all groups, medical students, dental students, pharmacy students, nursing students) and health personnel (all groups, medical staff, dentists, pharmacists, nurses).

Intervention: E-cigarette.

Comparator: Conventional cigarette (for prevalence only).

Study designs to be included: Observational studies, mainly crosssectional studies.

Eligibility criteria: (a) Study participants are health sciences students and health professionals on any age, gender, courses, and occupational groups. (b) Studies that provide data on prevalence of usage of conventional and electronic cigarettes. (c) Studies that provide data on knowledge and attitude toward e-cigarette or vaping.
Information sources: An extensive literature search using a combination of mesh terms (Electronic Nicotine Delivery Systems) and text words (e-cigarette, electronic cigarette, vaping) will be carried out on electronic databases such as PubMed and Scopus. This will be supplemented by a Google Scholar search using the same search terms mentioned above. The year of publication of included studies will be from Jan 2017 to Dec 2020

Main outcome(s): Prevalence of e-cigarette use health sciences students and health personnel.

Additional outcome(s): (a) Knowledge and attitude of health sciences students and health personnel regarding e-cigarette; (b) Prevalence of conventional cigarette use health sciences students and health personnel.

Data management: References retrieved will be managed used citation manager Endnote 7.

Quality assessment / Risk of bias analysis: The studies identified will then be assessed for methodological validity and relevant data in the included studies were extracted by a pair of investigators and checked by a senior researcher, independently, to minimize potential bias and error as well as to resolve any disagreements. The quality assessment of the published studies will be assessed using Newcastle Ottawa Scale.

Strategy of data synthesis: For quantitative data, where possible, meta-analysis will be performed using MedCalc Statistical Software using fixed effect model if study

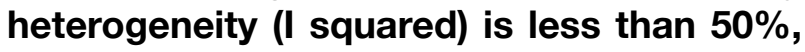
otherwise random effect model will be used.

Subgroup analysis: Where possible, prevalence rate may be stratified by WHO regions.

Sensitivity analysis: None.

Language: English. 
Country(ies) involved: Malaysia.

Other relevant information: This systematic review will be prepared following MOOSE guidelines.

Keywords: Electronic Nicotine Delivery Systems; Smoking; Health Knowledge, Attitude and Practice; Prevalence; health sciences students; Health Personnel.

Dissemination plans: One journal article is planned.

Contributions of each author:

Author 1 - Cheong Lieng Teng - Develop the proposal, supervise data retrieval and management, conduct data synthesis, manuscript writing.

Email: tengcl@gmail.com

Author 2 - Asiya Begum Vasiwala - Develop the proposal, data retrieval and management, manuscript writing.

Email: ashiya1096@gmail.com

Author 3 - Heyman Raj Bari - Develop the proposal, data retrieval and management, manuscript writing.

Email: hymnraj@gmail.com

Author 4 - Erwin Jiayuan Khoo - Develop the proposal, supervise data retrieval and management, manuscript writing.

Email: jiayuan_khoo@imu.edu.my 